9-1993

\title{
Semiclassical Approach to Quantum-mechanical Problems with Broken Supersymmetry
}

\author{
Ranabir Dutt \\ Visva Bharati University \\ Asim Gangopadhyaya \\ Loyola University Chicago, agangop@luc.edu \\ Avinash Khare \\ Sachivalaya Marg

\begin{abstract}
A. Pagnamenta
University of Illinois at Chicago

Uday P. Sukhatne

University of Illinois at Chicago, sukhatme@uic.edu
\end{abstract}

Follow this and additional works at: https://ecommons.luc.edu/physics_facpubs

Part of the Physics Commons

\section{Recommended Citation}

Dutt, R., A. Gangopadhyaya, Avinash Khare, A. Pagnamenta, and U. Sukhatme. "Semiclassical Approach to Quantum-Mechanical Problems with Broken Supersymmetry." Physical Review A 48, no. 3 (September 1, 1993): 1845-53. doi:10.1103/PhysRevA.48.1845.

This Article is brought to you for free and open access by the Faculty Publications and Other Works by Department at Loyola eCommons. It has been accepted for inclusion in Physics: Faculty Publications and Other Works by an authorized administrator of Loyola eCommons. For more information, please contact ecommons@luc.edu.

\section{(c) (i) $\Theta$}

This work is licensed under a Creative Commons Attribution-Noncommercial-No Derivative Works 3.0 License. (C) 1993 The American Physical Society. 


\title{
Semiclassical approach to quantum-mechanical problems with broken supersymmetry
}

\author{
R. Dutt \\ Department of Physics, Visva-Bharati University, Santiniketan 731235, West Bengal, India \\ A. Gangopadhyaya \\ Department of Physics, Loyola University Chicago, Chicago, Illinois 60626 \\ Avinash Khare \\ Institute of Physics, Sachivalaya Marg, Bhubaneswar 751005, India \\ A. Pagnamenta and U. Sukhatme \\ Physics Department, University of Illinois at Chicago, Chicago, Illinois 60680
}

(Received 8 December 1992)

\begin{abstract}
The semiclassical WKB approximation method is reexamined in the context of nonrelativistic quantum-mechanical bound-state problems with broken supersymmetry (SUSY). This gives rise to an alternative quantization condition (denoted by BSWKB) which is different from the standard WKB formula and also different from the previously studied supersymmetric (SWKB) formula for unbroken SUSY. It is shown that to leading order in $\hbar$, the BSWKB condition yields exact energy eigenvalues for shape-invariant potentials with broken SUSY (harmonic oscillator, Pöschl-Teller I and II) which are known to be analytically solvable. Further, we show explicitly that the higher-order corrections to these energy eigenvalues, up to sixth order in $\hbar$, vanish identically. We also consider a number of examples of potentials with broken supersymmetry that are not analytically solvable. In particular, for the broken SUSY superpotential $W=A x^{2 d},[A>0, d=$ (integer)], we evaluate contributions up to the sixth order and show that these results are in excellent agreement with numerical solutions of the Schrödinger equation. While the numerical BSWKB results in lowest order are not always better than the corresponding WKB results, they are still a considerable improvement because they guarantee equality of the corresponding energy eigenvalues for the supersymmetric partner potentials $V_{+}$and $V_{-}$. This is of special importance in those situations where these partner potentials are not related by parity.

PACS number(s): 03.65.Sq, 03.65.Ge, 11.30.Pb
\end{abstract}

\section{INTRODUCTION}

During the past few years there has been a revival of interest in semiclassical approximation schemes as applied to nonrelativistic quantum-mechanical problems. In 1985, using the framework of supersymmetric quantum mechanics (SUSYQM) [1], Comtet, Bandrauk, and Campbell [2] proposed a leading-order quantization condition similar to the well-known WKB formula [3-5] for the case where supersymmetry is unbroken. This formula, which we refer to as the SWKB quantization condition, was shown to provide exact spectra [6-9] for all shape-invariant potentials [10]. Additional desirable features of the SWKB scheme are the following: the eigenvalue degeneracy relation of the unbroken supersymmetry (SUSY) is preserved by construction and, for spherically symmetric three-dimensional problems, the artificial Langer modification needed for restoring the threshold behavior of the WKB wave function is built in. By explicit calculation, Adhikari et al. [9] proved that corrections to the energy eigenvalues (up to sixth order in $\hbar$ ) are zero for all shape-invariant potentials. This obviously suggests that all higher-order corrections must also vanish. This was demonstrated for the Rosen-Morse potential by Raghunathan, Seetharaman, and Vasan [11]. Recently, Barclay and Maxwell [12] proved quite generally that shape-invariant potentials have zero higherorder SWKB corrections. For non-shape-invariant potentials, for which exact analytic solutions are not obtainable, it has been observed [9] that, where the superpotential is available, better accuracy in predicting energy eigenvalues, compared to the usual WKB calculations $[4$, 5], is obtained by using the higher-order SWKB quantization condition [13]. However, all these calculations were concerned with examples of unbroken supersymmetry.

Relatively little attention has been paid so far to study problems involving broken SUSY. This is perhaps due to the fact that in the majority of examples cited in the literature, the supersymmetric partner potentials are related to each other by the parity operation since they are constructed from a symmetric superpotential and thus the energy degeneracy between the two partner potentials follows trivially. Furthermore, for the broken SUSY case, neither the ground-state energy nor the groundstate wave function are known in general. Chuan [14], Ralchenko and Semenov [15], and others [16,17] have 
discussed the possibility of finding connections between quantum-mechanical systems with broken and unbroken supersymmetry. Very recently we have made a critical study [18] of these aspects. We have been able to construct several analytically solvable examples of broken supersymmetry. Our examples are motivated by shapeinvariant potentials obeying unbroken SUSY [19-21] and are obtained from them by changing the domains of the parameters which appear in the potentials via suitable mapping procedures.

In analogy to the situation of unbroken supersymmetry, one may ask at this point whether there exists a new quantization condition which reproduces the exact analytic eigenvalues for shape-invariant potentials with broken SUSY. If so, one might further expect that higherorder corrections to the leading-order term should also vanish as is the case for unbroken supersymmetry. In this paper, we examine these possibilities in detail. To the best of our knowledge, no elaborate investigation of broken supersymmetric WKB (BSWKB) calculations has been made so far, except the work of Inomata and Junker [22] in which the leading-order BSWKB quantization condition was obtained from a path integral approach. However, a difficulty with their approach is that it is quite complicated to derive higher-order terms explicitly. In this paper, we follow the method given in Ref. $[9]$.

In Sec. II we give a summary of the algebraic structure of SUSYQM. Specifically, we discuss conditions on the superpotential which lead to broken supersymmetry. In Sec. III our calculation of the semiclassical quantization condition up to sixth order for broken supersymmetry is presented. (We only consider the usual situation of two classical turning points.) In Sec. IV we show that the leading-order BSWKB quantization condition yields exact energy eigenvalues for the known shape-invariant potentials with broken SUSY (harmonic oscillator, PöschlTeller I and II) [18]. We also demonstrate that the higherorder corrections to the leading-order energy eigenvalues, up to sixth order in $\hbar$, vanish identically. It is important to notice that our scheme preserves the energy degeneracy relation for the partner potentials at each order of the BSWKB approximation. This is not the case for the higher-order standard WKB method. Our numerical analyses for three broken SUSY non-shape-invariant potentials are presented in Sec. V.

For these potentials, we have compared the results from BSWKB and WKB approximations, both in lowest order, and we find that with the noted exception of the ground state, BSWKB does much better than the usual WKB method. For a superpotential of the form $W=A x^{2 d}(A>0, d=1)$ the BSWKB contributions to order $\hbar^{6}$ have been computed analytically. These results are found to be in excellent agreement with numerical solutions of the Schrödinger equation. Concluding remarks are presented in Sec. VI.

\section{SUPERSYMMETRIC QUANTUM MECHANICS}

Supersymmetric quantum mechanics is well developed by now [23]. According to Witten [1], SUSYQM is char- acterized by the supercharge operators $Q_{i}(i=1,2, \ldots, N)$ which are self-adjoint (i.e., $Q_{i}=Q_{i}^{\dagger}$ ) and satisfy the following graded algebra:

$$
\left[Q_{i}, H\right]=0,\left\{Q_{i}, Q_{j}\right\}=H \delta_{i j},
$$

where $H$ is the supersymmetric Hamiltonian. The algebra implies that the spectrum of $H$ is positive semidefinite. For the understanding of unbroken and/or broken supersymmetry, we consider the simplest nontrivial case, $N=2$. In terms of $Q=\left(Q_{1}+i Q_{2}\right) / \sqrt{2}$ and its adjoint $Q^{\dagger}=\left(Q_{1}-i Q_{2}\right) / \sqrt{2}$, the algebra governing this supersymmetric system is given by

$$
H=\left\{Q, Q^{\dagger}\right\}, Q^{2}=0, \quad\left(Q^{\dagger}\right)^{2}=0 .
$$

Obviously,

$$
[Q, H]=\left[Q^{\dagger}, H\right]=0 .
$$

For SUSY to be a good symmetry it is necessary that

$$
Q|0\rangle=Q^{\dagger}|0\rangle=0,
$$

where $|0\rangle$ denotes the ground state.

A simple realization of the algebra defined by Eq. (2) can be achieved by considering

$$
Q=\left(\begin{array}{ll}
0 & 0 \\
A & 0
\end{array}\right), \quad Q^{\dagger}=\left(\begin{array}{cc}
0 & A^{\dagger} \\
0 & 0
\end{array}\right),
$$

where $A$ is a linear operator and $A^{\dagger}$ is its adjoint:

$$
A=\frac{\hbar}{\sqrt{2 m}} \frac{d}{d x}+W(x), \quad A^{\dagger}=-\frac{\hbar}{\sqrt{2 m}} \frac{d}{d x}+W(x) .
$$

Equations (2)-(6) lead to the supersymmetric Hamiltonian

$$
H=\left(\begin{array}{cc}
H_{-} & 0 \\
0 & H_{+}
\end{array}\right)
$$

with

$$
\begin{aligned}
& H_{-}=A^{\dagger} A=-\frac{\hbar^{2}}{2 m} \frac{d^{2}}{d x^{2}}+V_{-}(x), \\
& H_{+}=A A^{\dagger}=-\frac{\hbar^{2}}{2 m} \frac{d^{2}}{d x^{2}}+V_{+}(x) \\
& V_{ \pm}(x)=W^{2}(x) \pm \frac{\hbar}{\sqrt{2 m}} W^{\prime}(x)
\end{aligned}
$$

The potentials $V_{+}$and $V_{-}$are called supersymmetric partner potentials and $W(x)$ is referred to as the superpotential.

The eigenstates of the Hamiltonian $H$ are doublets

$$
\psi_{n}(x)=\left(\begin{array}{c}
\psi_{n}^{(-)} \\
\psi_{n}^{(+)}
\end{array}\right)
$$

where $\psi_{n}^{( \pm)}$satisfy the eigenvalue equations

$$
H_{-} \psi_{n}^{(-)}=E_{n}^{(-)} \psi_{n}^{(-)}, \quad H_{+} \psi_{n}^{(+)}=E_{n}^{(+)} \psi_{n}^{(+)} .
$$


If $E_{0}^{(-)}=0$ is an eigenvalue of $H_{-}$, then $A \psi_{0}^{(-)}=0$. It then follows from Eq. (6) that

$$
\psi_{0}^{(-)}=\exp \left[-\sqrt{2 m} \hbar \int^{x} W\left(x^{\prime}\right) d x^{\prime}\right] .
$$

If SUSY is an unbroken symmetry, one wants $\psi_{0}^{(-)}$to be an acceptable wave function. It must be quadratically integrable and satisfy the correct boundary conditions, that is, it must vanish at $x_{L}$ and $x_{R}$ which denote the left and right end points of the variable $x$. In this case one gets the energy degeneracy relation

$$
E_{n+1}^{(-)}=E_{n}^{(+)}
$$

On the other hand, if $1 / \psi_{0}^{(-)}$vanishes at the end points, then the roles of $V_{-}$and $V_{+}$are reversed. Consequently, the ground-state energy of $V_{+}$, i.e., $E_{0}^{(+)}=0$, whereas now $E_{0}^{(-)} \neq 0$.

If neither $\psi_{0}^{(-)}$nor $1 / \psi_{0}^{(-)}$vanish at the end points, that is, neither $V_{-}$nor $V_{+}$has a zero energy bound state, we have the situation of broken SUSY. From here on we consider only cases where $W(x)$ is nonzero and of definite sign as $x \rightarrow x_{L}$ and as $x \rightarrow x_{R}$. For unbroken SUSY to hold, the boundary conditions require the wave function $\psi_{0}^{(-)}$to vanish at both ends. This happens in Eq. (13) if $W(x)$ is such that $I_{R} \equiv \int_{x_{0}}^{x_{R}} W(x) d x=+\infty$ and $I_{L} \equiv \int_{x_{L}}^{x_{0}} W(x) d x=-\infty$ for any $x_{0}$ such that $x_{L}<x_{0}<x_{R}$. Under our assumptions this can only hold if $W\left(x_{L}\right)$ and $W\left(x_{R}\right)$ are of opposite sign.

However, even if $W\left(x_{L}\right)$ and $W\left(x_{R}\right)$ are of opposite sign, unbroken SUSY is not guaranteed, since $I_{R}$ or $I_{L}$ may be finite. In this case $\psi_{0}^{(-)}$does not satisfy the boundary conditions and one has broken SUSY. However, if $W\left(x_{L}\right)$ and $W\left(x_{R}\right)$ have the same sign one definitely has broken SUSY. In this case, the spectra of both $H_{-}$ and $H_{+}$begin at positive values and are degenerate, that is,

$$
E_{n}^{(-)}=E_{n}^{(+)}
$$

\section{THE BSWKB QUANTIZATION CONDITION}

Some time ago, Adhikari et al. [9] have derived the SWKB quantization condition to order $\hbar^{6}$ in the case where SUSY is unbroken. In this section, we follow their method but only indicate the steps that require modification in the situation where SUSY is broken.

Let us start with the Schrödinger equation

$$
\left[-\frac{\hbar^{2}}{2 m} \frac{d^{2}}{d x^{2}}+V(x)-E\right] \psi(x)=0
$$

for any one-dimensional potential $V(x)$. We only consider the usual case of two classical turning points $x_{1}$ and $x_{2} \cdot\left(\geq x_{1}\right)$ given by

$$
E-V(x)=0 \text {. }
$$

On inserting the WKB wave function

$$
\psi(x)=\exp \left(\frac{i}{\hbar} \int^{x} S(y) d y\right)
$$

into Eq. (16) and following the steps of Ref. [9], one can show that the WKB quantization condition to order $\hbar^{6}$ is given by [3-5]

$$
\begin{aligned}
& \sqrt{2 m} \int_{x_{1}}^{x_{2}}(E-V)^{\frac{1}{2}} d x-\frac{\hbar^{2}}{24 \sqrt{2 m}} \frac{d}{d E} \int_{x_{1}}^{x_{2}} V^{\prime \prime}(E-V)^{-\frac{1}{2}} d x \\
& +\frac{\hbar^{4}}{2880(2 m)^{3 / 2}}\left\{\frac{d^{3}}{d E^{3}} \int_{x_{1}}^{x_{2}}\left[7\left(V^{\prime \prime}\right)^{2}-5 V^{\prime} V^{\prime \prime \prime}\right](E-V)^{-\frac{1}{2}} d x\right\} \\
& -\frac{\hbar^{6}}{725760(2 m)^{5 / 2}}\left\{\frac{d^{4}}{d E^{4}} \int_{x_{1}}^{x_{2}} 216\left(V^{\prime \prime \prime}\right)^{2}(E-V)^{-\frac{1}{2}} d x\right. \\
& \left.\quad+\frac{d^{5}}{d E^{5}} \int_{x_{1}}^{x_{2}}\left[93\left(V^{\prime \prime}\right)^{3}-224 V^{\prime} V^{\prime \prime} V^{\prime \prime \prime}+35\left(V^{\prime}\right)^{2} V^{\prime \prime \prime \prime}\right](E-V)^{-\frac{1}{2}} d x\right\}=\left(n+\frac{1}{2}\right) \pi \hbar .
\end{aligned}
$$

We begin with this form of the WKB quantization condition and obtain an alternative series expansion in the BSWKB approximation. In particular, we apply Eq. (19) to the SUSY potential $V_{-}(x)$ as given in Eq. (10). On substituting $W^{2}(x)-\frac{\hbar}{\sqrt{2 m}} W^{\prime}(x)$ in place of $V$ in Eq. (19) and expanding the left-hand side in powers of $\hbar$ one obtains

$$
\begin{gathered}
\sqrt{2 m} \int_{a}^{b} \sqrt{E_{n}^{(-)}-W^{2}} d x+\frac{\hbar}{2} \int_{a}^{b} \frac{W^{\prime}(x) d x}{\sqrt{E_{n}^{(-)}-W^{2}}}+\ldots \\
=\left(n+\frac{1}{2}\right) \pi \hbar,
\end{gathered}
$$

where the turning points $a, b$ are the solutions of the equa- 
tion

$$
E_{n}^{(-)}-W^{2}(x)=0
$$

Note that the turning points, $x_{1}$ and $x_{2}$, of the $V_{-}$partner potential are different from $a$ and $b$ since they are the solutions of the equation

$$
E_{n}^{(-)}-W^{2}(x)+\frac{\hbar}{\sqrt{2 m}} W^{\prime}(x)=0 .
$$

It is worth noting that in obtaining the higher-order BSWKB integrals one would expect contributions to different orders in $\hbar$ due to the change of the limits from $x_{1}, x_{2} \rightarrow a, b$, as well as from terms coming from the expansion of the integrands in powers of $\hbar$. Following Ref. [9], one can show that the contributions due to the change in the limits cancel in the $\hbar$ expansion.

The order $\hbar$ term on the left-hand side of Eq. (20) can be integrated immediately to yield

$$
\left.\frac{\hbar}{2} \sin ^{-1}\left(\frac{W(x)}{\sqrt{E_{n}}}\right)\right|_{a} ^{b} .
$$

It is at this stage that the difference between broken and unbroken SWKB manifests itself. We now assume that $W(x)$ retains the same sign from the left classical turning point $a$ out to $x_{L}$ and from the right classical turning point $b$ out to $x_{R}$. This is reasonable since a change of sign in $W$ would introduce additional oscillations in $W^{2}$, resulting in more than two turning points. In the unbroken SUSY case $W(x)$ has opposite signs at the two turning points

$$
W(a)=-W(b)=-\sqrt{E_{n}} .
$$

However, if $W(x)$ has the same sign at the two turning points, this corresponds to the broken SUSY case, and

$$
W(a)=W(b)= \pm \sqrt{E_{n}},
$$

where both can be positive or both negative (see our examples below). As a result, unlike in the unbroken case, the order $\hbar$ contribution in the BSWKB integral Eq. (20) vanishes. One can easily show that, apart from this crucial difference, all higher-order terms in the $\hbar$ expansion in broken and in unbroken SWKB are identical. Following Ref. [9], the BSWKB quantization condition to order $\hbar^{6}$ is given by (note that here $E$ corresponds to $E_{n}^{(-)}$)

$$
\begin{aligned}
& (2 m)^{\frac{1}{2}} \int_{a}^{b}\left(E-W^{2}\right)^{\frac{1}{2}} d x-\frac{\hbar^{2} E}{6(2 m)^{\frac{1}{2}}} \frac{d^{2}}{d E^{2}} \int_{a}^{b}\left(W^{\prime}\right)^{2}\left(E-W^{2}\right)^{-\frac{1}{2}} d x \\
& +\frac{\hbar^{4}}{720(2 m)^{3 / 2}}\left\{\frac{d^{2}}{d E^{2}} \int_{a}^{b} 30 W^{\prime} W^{\prime \prime \prime}\left(E-W^{2}\right)^{-\frac{1}{2}} d x\right. \\
& \left.+\frac{d^{3}}{d E^{3}} \int_{a}^{b}\left[-8\left(W^{\prime}\right)^{4}-31\left(W^{\prime}\right)^{2} W W^{\prime \prime}+7 W^{2}\left(W^{\prime \prime}\right)^{2}-5 W^{2} W^{\prime} W^{\prime \prime \prime}\right]\left(E-W^{2}\right)^{-\frac{1}{2}} d x\right\} \\
& +\frac{\hbar^{6}}{90720(2 m)^{5 / 2}}\left\{\frac{d^{3}}{d E^{3}} \int_{a}^{b} 378\left(W^{\prime \prime \prime}\right)^{2}\left(E-W^{2}\right)^{-\frac{1}{2}} d x\right. \\
& +\frac{d^{4}}{d E^{4}} \int_{a}^{b}\left[-2160 W^{\prime} W^{\prime \prime} W^{\prime \prime \prime}+1674\left(W^{\prime}\right)^{2}\left(W^{\prime \prime}\right)^{2}-108 W^{2}\left(W^{\prime \prime \prime}\right)^{2}\right]\left(E-W^{2}\right)^{-\frac{1}{2}} d x \\
& +\frac{d^{5}}{d E^{5}} \int_{a}^{b}\left[96\left(W^{\prime}\right)^{6}-1119 W^{\prime}\left(W^{\prime}\right)^{4} W^{\prime \prime}+729 W^{2}\left(W^{\prime}\right)^{2}\left(W^{\prime \prime}\right)^{2}+399 W^{2}\left(W^{\prime}\right)^{3} W^{\prime \prime \prime}\right.
\end{aligned}
$$

Thus the only difference between the broken and the unbroken SWKB quantization schemes is the right-hand side term $\left(n+\frac{1}{2}\right) \pi \hbar$ in the broken case and $n \pi \hbar$ in the unbroken scheme, while the left-hand sides in both cases are identical.

It is worth pointing out here that if instead of $E_{n}^{(-)}$, one is interested in obtaining a quantization formula for $E_{n}^{(+)}$[i.e., the energy eigenvalues of the SUSY partner potential $\left.V_{+}(x)\right]$, one needs to replace $\frac{\hbar}{\sqrt{2 m}} W^{\prime}(x)$ by $-\frac{\hbar}{\sqrt{2 m}} W^{\prime}(x)$, which is equivalent to replacing $\hbar$ by $-\hbar$ in the left-hand side of Eq. (26). It immediately follows that, unlike the unbroken case, the BSWKB quantization condition for $E_{n}^{(+)}$is also given by the same formula (26), proving that all higher-order BSWKB calculations will also retain the degeneracy relation, Eq. (15), which is a consequence of broken supersymmetry.

For exactly the same reason as in the SWKB scheme, in the BSWKB quantization scheme one also obtains the correct threshold behavior of the radial wave function for spherically symmetric problems without the need for 
any Langer-type modifications [9]. In that sense both the broken and the unbroken SWKB schemes are superior to the WKB approach.

\section{APPLICATION TO BROKEN SUSY SHAPE-INVARIANT POTENTIALS}

How good is the BSWKB quantization scheme? To that end we apply the quantization condition, Eq. (26), to the three analytically solvable shape-invariant potentials with broken SUSY. Using units where $\hbar=2 m=1$, the corresponding superpotentials are [18] as follows.

Harmonic oscillator:

$$
\left.\begin{array}{r}
W=\omega r / 2-(l+1) / r \\
0 \leq r<\infty
\end{array}\right\} \begin{aligned}
& \omega<0, l>0 \\
& \omega>0, l<-2
\end{aligned}
$$

Pöschl-Teller I:

$$
\begin{aligned}
& W_{\mathrm{PTI}}(A, B, \alpha)=A \tan (\alpha x)-B(\cot \alpha \mathrm{x}) \mid A<-1, B>1 \\
& 0<x<\pi / 2\} A>1, B<-1 \text {. }
\end{aligned}
$$

Pöschl-Teller II:

$$
\begin{aligned}
& \left.W_{\text {PTII }}(A, B, \alpha)=A \tanh (\alpha r)-B \operatorname{coth}(\alpha r)\right\} A+B+1<0, B>1 \\
& 0 \leq r<\infty\} A+B-1>0, B<-1 \text {. }
\end{aligned}
$$

The indicated conditions on the different parameters assure broken SUSY and the existence of degenerate energy levels for the partner potentials $V_{+}$and $V_{-}$. Because the last potential has a finite well height, only a finite number of eigenstates are allowed for given values of $A$ and $B$. We should perhaps remark here that among all known shape-invariant potentials, these are the only examples of broken SUSY [18] where both $V_{-}$and $V_{+}$simultaneously can hold bound states. The Scarf superpotential $W_{S}(A, B, \alpha) \equiv-A \cot (\alpha x)+B \operatorname{cosec}(\alpha x)$ and the superpotential $W_{T}(A, B, \alpha) \equiv-A \operatorname{coth}(\alpha r)+B \operatorname{cosech}(\alpha r)$, are also examples of broken SUSY, but can be shown to be equivalent to the Pöschl-Teller I ( $\left.W_{\mathrm{PTI}}\right)$ and Pöschl-Teller II ( $\left.\mathrm{W}_{\mathrm{PTII}}\right)$ superpotentials, respectively, by suitable redefinition of parameters. In particular,

$$
\begin{aligned}
W_{S}(A, B, \alpha) & \equiv-A \cot (\alpha x)+B \operatorname{cosec}(\alpha x) \\
& =\left(\frac{A+B}{2}\right) \tan \left(\frac{\alpha x}{2}\right)-\left(\frac{A-B}{2}\right) \cot \left(\frac{\alpha x}{2}\right) \\
& =W_{\text {PTI }}\left(\frac{A+B}{2}, \frac{A-B}{2}, \frac{\alpha}{2}\right) .
\end{aligned}
$$

Similarly,

$$
W_{T}(A, B, \alpha)=W_{\text {PTII }}\left(\frac{A+B}{2}, \frac{A-B}{2}, \frac{\alpha}{2}\right) .
$$

It turns out that in all three cases, the leading-order BSWKB quantization condition [which is just the first term on the left-hand side of Eq. (26), with $\hbar=2 m=1]$

$$
\int_{a}^{b} \sqrt{E-W^{2}} d x=\left(n+\frac{1}{2}\right) \pi
$$

yields exact energy eigenvalues, namely,

$$
E_{n}^{(+)}=E_{n}^{(-)}=\left\{\begin{array}{l}
-\omega(2 n+2 l+3) \text { for }(27 \mathrm{a}) \\
\omega(2 n-2 l-1) \text { for }(27 \mathrm{~b}) \\
(A-B-1-2 n)^{2}-(A+B)^{2} \text { for }(28 \mathrm{a}) \\
(A-B+1+2 n)^{2}-(A+B)^{2} \text { for }(28 \mathrm{~b}) \\
-(A+B+2 n+1)^{2}+(A-B)^{2} \text { for }(29 \mathrm{a}) \\
-(A+B-2 n-1)^{2}+(A-B)^{2} \text { for }(29 \mathrm{~b})
\end{array}\right.
$$

The above energies correspond to the simple choice $\alpha=1$ in $W_{\mathrm{PTI}}$ and $W_{\mathrm{PTII}}$. We have explicitly computed the order $\hbar^{2}$ to $\hbar^{6}$ correction terms as given by Eq. (26) and find that for all the above superpotentials these corrections vanish identically.

Before ending this section, we would like to point out one curious fact about the three shape-invariant potentials given by Eqs. (27)-(29). It turns out that in all three cases, the corresponding potential can be equally well described by either unbroken or broken SUSY and the corresponding lowest-order SWKB or BSWKB approximations yield exact eigenvalues. For example, the 
Pöschl-Teller I superpotential $W_{\mathrm{PTI}}(A, B, \alpha=1)=$ $A \tan x-B \cot x$ gives the potential

$$
V_{-}(x)=A(A-1) \sec ^{2} x+B(B-1) \operatorname{cosec}^{2} x-(A+B)^{2} .
$$

For $A>1, B>1$, we have unbroken SUSY and the exact energy eigenvalues are [17]

$$
E_{n}^{(-)}=(A+B+2 n)^{2}-(A+B)^{2},
$$

which can be obtained analytically. The SWKB condition also gives the exact answer. as

Alternatively, the potential $V_{-}(x)$ can also be rewritten

$$
V_{-}(x)=\tilde{V}_{-}(x)+\left[(A+\tilde{B})^{2}-(A-\tilde{B}+1)^{2}\right],
$$

where

$$
\begin{gathered}
\tilde{V}_{-}(x)=A(A-1) \sec ^{2} x+\tilde{B}(\tilde{B}-1) \operatorname{cosec}^{2} x-(A+\tilde{B})^{2}, \\
\tilde{B}=1-B .
\end{gathered}
$$

Clearly, $\tilde{V}_{-}(x)$ corresponds to the broken SUSY PöschlTeller I superpotential given in Eq. (28b), since $A>1$ and $\tilde{B}<0$. Its eigenvalues are $\tilde{E}_{n}=(A-B+2 n+$ $1)^{2}-(A+\tilde{B})^{2}$, and as discussed before, can be obtained analytically or from the BSWKB quantization condition. Using Eq. (33) and $\tilde{E}_{n}$, one gets the eigenvalues $E_{n}$ given in Eq. (32). A similar treatment [18] is also applicable to the other two shape-invariant potentials given in Eqs. (27) and (29). It is intriguing that for all three broken SUSY shape-invariant potentials [Eqs. (27)-(29)], both broken as well as unbroken lowest-order SWKB formulas reproduce the exact bound-state spectrum (while WKB does not do so). A deeper reason for this presumably does exist but as of now it has not been brought out. Note that even though the eigenvalues are reproduced exactly, semiclassical wave functions are known to be not exact [24-27]. In particular, they diverge at the classical turning points. Also, the transmisson and reflection coefficients $T$ and $R$ are not fully given by the semiclassical methods [28].

\section{NON-SHAPE-INVARIANT POTENTIALS WITH BROKEN SUSY}

In the preceding section, we have discussed three shape-invariant potentials with parameters consistent with broken SUSY conditions. In this section, we deal with a few non-shape-invariant potentials which do not admit exact analytic solutions. First, we give a simple one-dimensional example where the partner potentials are related to each other by a parity operation. This trivially implies that the energy eigenvalues of the partner potentials are identical. For this example we give the broken SUSY quantization formula explicitly to sixth order in $\hbar$. Our second one-dimensional potential leads to partner potentials which are no longer related to each other by parity and the degeneracy of the spectra becomes a nontrivial consequence of broken SUSY. For the third example we have selected a spherically symmetric superpotential. Again, the degeneracy of the spectra of the partners is an interesting consequence of broken SUSY.

To begin with, let us consider the broken SUSY superpotential

$$
W(x)=A x^{2 d},
$$

which generates the supersymmetric partner potentials

$$
V_{ \pm}=A^{2} x^{4 d} \pm 2 A d \frac{\hbar}{\sqrt{2 m}} x^{(2 d-1)} .
$$

From Fig. 1, one may note that these partner potentials are related to each other by parity transformations and consequently the degeneracy of the energy eigenvalues $E_{n}^{(-)}=E_{n}^{(+)}$is trivially guaranteed. Using (35) in (26) and evaluating the integrals explicitly, one obtains for the energy eigenvalues

$$
E_{n}=\left[A^{1 / d} G_{n}^{2} /(2 m)\right]^{\frac{2 d}{2 d+1}},
$$

where $G_{n}$ is given by the following equation which, for given values of $d$ and the quantum number $n$, can be solved numerically

$$
\begin{aligned}
G_{n} \sqrt{\pi} & \frac{\Gamma\left(\frac{4 d+1}{4 d}\right)}{\Gamma\left(\frac{6 d+1}{4 d}\right)}+\hbar^{2} \sqrt{\pi} \frac{(2 d+1)}{12 G_{n}} \frac{\Gamma\left(\frac{4 d-1}{4 d}\right)}{\Gamma\left(\frac{2 d-1}{4 d}\right)}-\hbar^{4} \sqrt{\pi} \frac{(2 d+3)}{1440 G_{n}^{3}} \frac{\Gamma\left(\frac{4 d-3}{4 d}\right)}{\Gamma\left(\frac{2 d-3}{4 d}\right)} \\
\times & {\left[60(2 d-1)(d-1)+\frac{(4 d-3)}{2 d}\left(-16 d^{2}-31 d(2 d-1)+\frac{7}{2}(2 d-1)^{2}-5(2 d-1)(d-1)\right)\right] } \\
+ & \hbar^{6} \sqrt{\pi} \frac{(2 d+5)(6 d+5)}{2903040 d^{2} G_{n}^{5}} \frac{\Gamma\left(\frac{4 d-5}{4 d}\right)}{\Gamma\left(\frac{2 d-5}{4 d}\right)} \\
\times & {\left[6048 d(2 d-1)^{2}(d-1)^{2}+54(2 d-1)^{2}(4 d-5)\left\{-160 d(d-1)+124 d^{2}-8(d-1)^{2}\right\}\right.} \\
& +(8 d-5)(4 d-5)\left(384 d^{3}-2238 d^{2}(2 d-1)+729 d(2 d-1)^{2}\right. \\
& \quad+798 d(d-1)(2 d-1)-\frac{93}{2}(2 d-1)^{3}+224(d-1)(2 d-1)^{2} \\
& -35(d-1)(2 d-1)(2 d-3))]=\left(n+\frac{1}{2}\right) \pi \hbar .
\end{aligned}
$$


It is amusing to note that the left-hand side of Eq. (38) can be simply obtained from Eq. (4.3) of Ref. [9] by the replacement of $d$ by $d-\frac{1}{2}$. This is because the higher-order corrections in both SWKB and in BSWKB are formally the same and in Ref. [9] the superpotential $W=A x^{2 d+1}$, corresponding to unbroken SUSY, was considered.

Since, for these potentials, the Schrödinger equation cannot be solved analytically, we compare the BSWKB energy eigenvalues with numerical results which we have obtained from the Schrödinger equations using a RungeKutta integration routine. In all our numerical work we use units in which $\hbar=2 m=1$. Therefore in all our figures and tables we give dimensionless quantities $E, V$, and $x$ (or $r$ ). These quantities can then be obtained in any desired units by multiplying $V$ and $E$ by the factor $\hbar^{2} /\left(2 m r_{0}^{2}\right)$, where $r_{0}$ is the desired unit of length.

For concreteness, we take $A=1$, and $d=1$ in Eq. (35). Our results are presented in Table I. For numerical comparisons, we also show the percentage errors, defined as $100 \times[E($ approx $)-E($ num $)] / E($ num $)$. First we compare our leading-order BSWKB values with the leadingorder WKB method. The tabulated results indicate that, except for the ground state, the lowest-order BSWKB eigenvalues are of comparable accuracy as the standard WKB eigenvalues. In Table I, we have given all entries to three places after the decimal, which is enough to indicate the differences with lowest-order WKB results which is all that we have available. The higher-order BSWKB results are of very high accuracy. The percentage errors, also given in the table, show that the SWKB values for

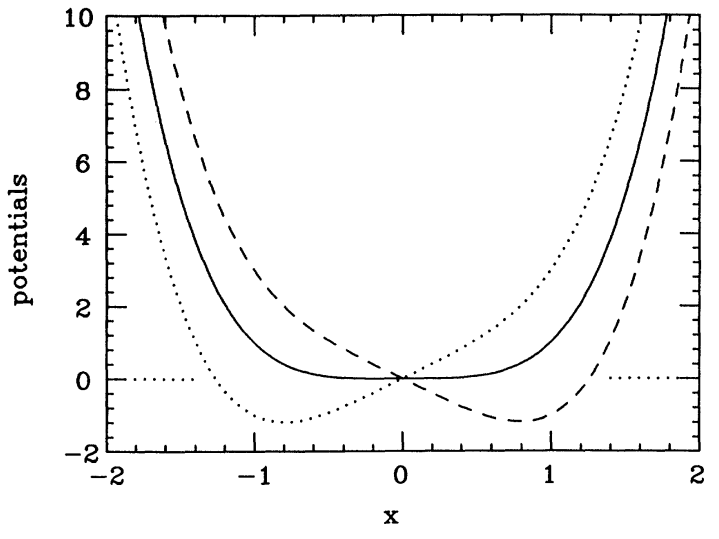

FIG. 1. The potentials $V_{+}$(dotted), $V_{-}$(dashed), $W^{2}$ (solid) associated with the superpotential $W=$ $A x^{2 d}, A=1, d=1$. The figure clearly shows how the two partner potentials are related by a parity operation and therefore trivially have the same spectrum.

the higher-lying states $(n>2)$ are actually accurate to five places after the decimal point, an overall accuracy of seven digits. Also, the exact degeneracy of the spectra of $V_{+}$and of $V_{-}$does not follow from the standard WKB scheme, but is evident from our BSWKB formulation which preserves this degeneracy order by order. For this potential, the WKB values for $V_{+}$and for $V_{-}$are trivially equal because they are mirror images of each other. For the ground state, the WKB value is some-

TABLE I. Comparison of energy eigenvalues computed up to order $\hbar^{6}$ from the BSWKB quantization condition [Eq. (26)] with leading-order ordinary WKB results corresponding to the superpotential $W=A x^{2 d}, A=1, d=1$ in units of $2 m=\hbar=1$. The last column contains the values obtained by numerical integration of the Schrödinger equation. The numbers in parentheses below each value indicate the percentage error in comparison to the numerical value.

\begin{tabular}{|c|c|c|c|c|c|c|}
\hline \multirow[b]{2}{*}{$n$} & \multicolumn{4}{|c|}{ BSWKB } & WKB & \multirow[t]{2}{*}{ Numerical } \\
\hline & $O\left(\hbar^{0}\right)$ & $O\left(\hbar^{2}\right)$ & $O\left(\hbar^{4}\right)$ & $O\left(\hbar^{6}\right)$ & $O\left(\hbar^{0}\right)$ & \\
\hline 0 & $\begin{array}{c}0.867 \\
(56)\end{array}$ & $\begin{array}{c}0.731 \\
(32)\end{array}$ & $\begin{array}{c}0.848 \\
(53)\end{array}$ & $\begin{array}{c}0.963 \\
(74)\end{array}$ & $\begin{array}{c}0.522 \\
(-5)\end{array}$ & 0.554 \\
\hline 1 & $\begin{array}{c}3.752 \\
(1.159)\end{array}$ & $\begin{array}{c}3.692 \\
(-0.458)\end{array}$ & $\begin{array}{c}3.698 \\
(-0.297)\end{array}$ & $\begin{array}{c}3.699 \\
(-0.270)\end{array}$ & $\begin{array}{c}3.628 \\
(-2.184)\end{array}$ & 3.709 \\
\hline 2 & $\begin{array}{c}7.414 \\
(0.556)\end{array}$ & $\begin{array}{c}7.372 \\
(-0.014)\end{array}$ & $\begin{array}{c}7.373 \\
(0.000)\end{array}$ & $\begin{array}{c}7.373 \\
(0.000)\end{array}$ & $\begin{array}{c}7.329 \\
(-0.597)\end{array}$ & 7.373 \\
\hline 3 & $\begin{array}{l}11.612 \\
(0.285)\end{array}$ & $\begin{array}{c}11.578 \\
(-0.009)\end{array}$ & $\begin{array}{l}11.579 \\
(0.000)\end{array}$ & $\begin{array}{l}11.579 \\
(0.000)\end{array}$ & $\begin{array}{c}11.544 \\
(-0.302)\end{array}$ & 11.579 \\
\hline 4 & $\begin{array}{l}16.234 \\
(0.173)\end{array}$ & $\begin{array}{c}16.205 \\
(-0.006)\end{array}$ & $\begin{array}{l}16.206 \\
(0.000)\end{array}$ & $\begin{array}{l}16.206 \\
(0.000)\end{array}$ & $\begin{array}{c}16.177 \\
(-0.179)\end{array}$ & 16.206 \\
\hline 5 & $\begin{array}{c}21.214 \\
(0.118)\end{array}$ & $\begin{array}{l}21.189 \\
(0.000)\end{array}$ & $\begin{array}{c}21.189 \\
(0.000)\end{array}$ & $\begin{array}{l}21.189 \\
(0.000)\end{array}$ & $\begin{array}{c}21.164 \\
(-0.118)\end{array}$ & 21.189 \\
\hline 6 & $\begin{array}{l}26.506 \\
(0.083)\end{array}$ & $\begin{array}{c}26.484 \\
(0.000)\end{array}$ & $\begin{array}{c}26.484 \\
(0.000)\end{array}$ & $\begin{array}{c}26.484 \\
(0.000)\end{array}$ & $\begin{array}{c}26.462 \\
(-0.083)\end{array}$ & 26.484 \\
\hline 7 & $\begin{array}{c}32.078 \\
(0.062)\end{array}$ & $\begin{array}{c}32.058 \\
(0.000)\end{array}$ & $\begin{array}{c}32.058 \\
(0.000)\end{array}$ & $\begin{array}{c}32.058 \\
(0.000)\end{array}$ & $\begin{array}{c}32.038 \\
(-0.062)\end{array}$ & 32.058 \\
\hline 8 & $\begin{array}{c}37.904 \\
(0.048)\end{array}$ & $\begin{array}{c}37.886 \\
(0.000)\end{array}$ & $\begin{array}{c}37.886 \\
(0.000)\end{array}$ & $\begin{array}{c}37.886 \\
(0.000)\end{array}$ & $\begin{array}{c}37.867 \\
(-0.050)\end{array}$ & 37.886 \\
\hline 9 & $\begin{array}{c}43.964 \\
(0.039)\end{array}$ & $\begin{array}{l}43.947 \\
(0.000)\end{array}$ & $\begin{array}{l}43.947 \\
(0.000)\end{array}$ & $\begin{array}{l}43.947 \\
(0.000)\end{array}$ & $\begin{array}{c}43.929 \\
(-0.041)\end{array}$ & 43.947 \\
\hline 10 & $\begin{array}{c}50.240 \\
(0.032)\end{array}$ & $\begin{array}{c}50.224 \\
(0.000)\end{array}$ & $\begin{array}{c}50.224 \\
(0.000)\end{array}$ & $\begin{array}{c}50.224 \\
(0.000)\end{array}$ & $\begin{array}{c}50.208 \\
(-0.032)\end{array}$ & 50.224 \\
\hline
\end{tabular}




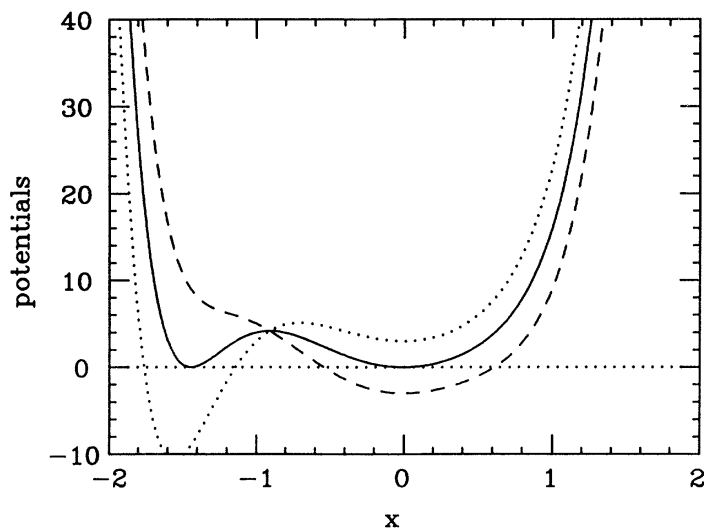

FIG. 2. The potentials $V_{+}$(dotted), $V_{-}$(dashed), $W^{2}$ (solid) associated with the superpotential $W=x^{4}+3 x$.

what better than the BSWKB value, but for this state none of the methods appears to work satisfactorily. This is not unexpected for a semiclassical approach.

The second superpotential (Fig. 2) which we consider is

$$
W=x^{4}+3 x .
$$

This superpotential is again positive at both end points $(x= \pm \infty)$ and obeys broken supersymmetry. It has the two partner potentials

$$
V_{ \pm}=x^{8}+6 x^{5} \pm 4 x^{3}+9 x^{2} \pm 3,
$$

which are not related to each other by a parity operation. The degeneracy of the spectra of these partner potentials is therefore a nontrivial consequence of broken supersymmetry.

We have evaluated the spectra of $V_{ \pm}$by three methods: using the broken SUSY quantization condition Eq. (26), by the usual WKB method using $V_{+}$and $V_{-}$separately, all in lowest-order only; and by numerical integration of the Schrödinger equation. The results are shown in Table II. We observe that for this potential the lowest-order

TABLE II. Energy eigenvalues corresponding to the asymmetric broken SUSY superpotential $W=x^{4}+3 x$, as obtained by the numerical method, the broken SUSY method, the WKB method for $V_{-}$, and the WKB method for $V_{+}$, all in leading order only.

\begin{tabular}{c|c|c|c|c}
\hline \hline$n$ & Numerical & BSWKB & WKB: $V_{-}$ & WKB: $V_{+}$ \\
\hline 0 & 0.362 & & & \\
1 & 6.121 & 6.09 & 5.96 & 6.18 \\
2 & 12.111 & 11.82 & 11.61 & 11.71 \\
3 & 19.159 & 19.14 & 18.91 & 18.97 \\
4 & 27.760 & 27.80 & 27.57 & 27.61 \\
5 & 37.620 & 37.66 & 37.43 & 37.46 \\
6 & 48.600 & 48.63 & 48.41 & 48.43 \\
7 & 60.667 & 60.65 & 60.43 & 60.45 \\
8 & 73.677 & 73.64 & 73.43 & 73.44 \\
9 & 87.656 & 87.57 & 87.36 & 87.38 \\
\hline \hline
\end{tabular}

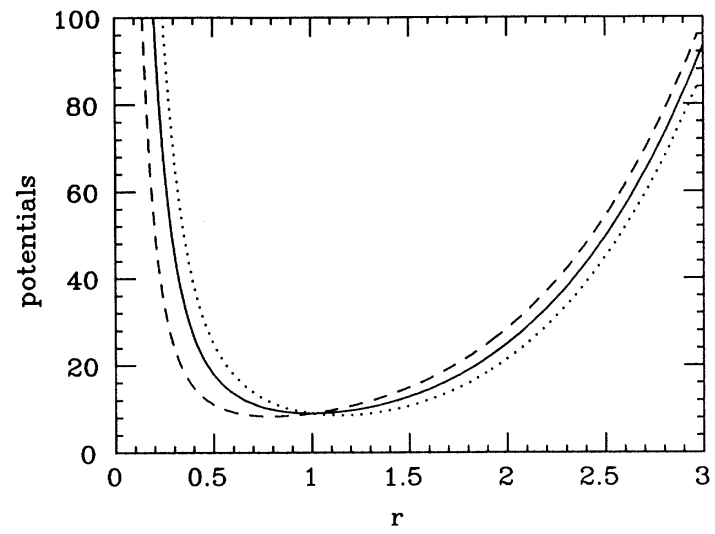

FIG. 3. The spherically symmetric potentials $V_{+}$(dotted), $V_{-}$(dashed), $W^{2}$ (solid) associated with the superpotential $W=-2 / r-r^{2}$. The spectra of the two partner potentials are degenerate, a nontrivial consequence of broken supersymmetry.

broken SUSY method is always more accurate than the WKB method, again with the exception of the ground state. For the ground state, $n=0$ (not shown in Table II) with a numerical eigenvalue of 0.362 , none of the semiclassical methods work. In fact, the broken SUSY method cannot even be applied to the ground state because this eigenvalue is in a region where the superpotential has four classical turning points.

Our third example (Fig. 3) of broken SUSY is the spherically symmetric superpotential

$$
W(r)=-2 / r-r^{2} .
$$

The results of numerical evaluation of the Schrödinger equation, use of the BSWKB method, and of the ordinary WKB method, all in lowest order only, are shown in Table III. It is noted that for this potential, again the broken SUSY method is more accurate than the standard WKB. While the broken SUSY method is consistently just slightly too high and gives the same values for both $V_{-}$and $V_{+}$, the WKB method for both $V_{-}$and for $V_{+}$is too low and gives differing values.

TABLE III. Energy eigenvalues for the partner potentials derived from the spherically symmetric superpotential $W=-2 / r-r^{2}$, as obtained by the numerical method, the broken SUSY method, the WKB method for $V_{-}$, and the WKB method for $V_{+}$.

\begin{tabular}{c|c|c|c|c}
\hline \hline$n$ & Numerical & BSWKB & WKB: $V_{-}$ & WKB: $V_{+}$ \\
\hline 0 & 13.34 & 13.46 & 12.85 & 13.07 \\
1 & 23.39 & 23.46 & 22.88 & 23.09 \\
2 & 34.58 & 34.64 & 34.05 & 34.27 \\
3 & 46.74 & 46.78 & 46.17 & 46.40 \\
4 & 59.73 & 59.76 & 59.13 & 59.37 \\
5 & 73.45 & 73.48 & 72.83 & 73.08 \\
6 & 87.84 & 87.86 & 87.19 & 87.45 \\
7 & 102.83 & 102.86 & 102.16 & 102.44 \\
\hline \hline
\end{tabular}




\section{CONCLUDING REMARKS}

In this paper, we have explicitly obtained the WKB quantization condition to order $\hbar^{6}$ for broken supersymmetric quantum-mechanical problems. Similar to the earlier observations for the SWKB quantization condition for the case of unbroken supersymmetry, our leadingorder BSWKB formula, Eq. (30), yields exact spectra for all shape-invariant potentials with broken SUSY and higher-order corrections turn out to be identically zero. We are not aware of any profound reason for this. A significant feature of the BSWKB formula, Eq. (26), as compared to the standard WKB formula is that it preserves the exact degeneracy relation, Eq. (15), at each order of the approximation. Note that for the potentials in Eqs. (40) and (41), the WKB predictions for $V_{+}$and $V_{-}$are indeed different. In this regard, the BSWKB method certainly has an advantage over the standard WKB method. Our numerical illustrations for three analytically not solvable broken SUSY potentials indicate that the lowest-order BSWKB quantization condition gives energy eigenvalues with accuracy comparable to the WKB predictions in lowest order. However, the inclusion of terms of higher order such as $\hbar^{4}$ and $\hbar^{6}$, which may only be possible because of the specific form of the $\mathrm{B}(\mathrm{SWKB})$ integrand, leads to almost exact results, which means that in these situations the BSWKB series is highly convergent. As expected for all semiclassical methods, the BSWKB results for highly excited states are substantially better than those for low-lying bound states. The BSWKB prediction for the ground-state energy is not that accurate in the three cases studied here. In fact, for our first potential, the BSWKB result to any order is even worse than the lowest-order $\mathrm{WKB}$ value. It is worth noting that the SWKB condition for unbroken SUSY guarantees the exact result $E_{0}^{(-)}=0$ by construction. However, a similar constraint is not available for the BSWKB quantization condition.

In order to be able to use the SWKB or the BSWKB quantization conditions, one needs to know the superpotential $W(x)$. Equivalently, if the ground-state wave function $\psi_{0}(x)$ or any other nodeless solution $\phi(x)$ of the Schrödinger equation for $\mathrm{V}_{-}$is known, then a nonsingular superpotential $W(x)$ is easily determined [29]. However, very often one only knows $V_{-}$. In this case the ordinary WKB method may be preferable since there is, in general, no easy way to determine $W(x)$.

\section{ACKNOWLEDGMENTS}

R.D., A.G. and A.K. would like to acknowledge the warm hospitality of the Physics Department of the University of Illinois at Chicago, where this work was done. This work was supported in part by the U. S. Department of Energy under Grant No. DE-FG02-84ER40173.
[1] E. Witten, Nucl. Phys. B185, 513 (1981); F. Cooper and B. Freedman, Ann. Phys. (N.Y.) 146, 262 (1983); C. V. Sukumar, J. Phys. A 18, 2917 (1985).

[2] A. Comtet, A. D. Bandrauk, and D. K. Campbell, Phys. Lett. 150B, 159 (1985); A. Khare, ibid. 161B, 131 (1985).

[3] J. L. Dunham, Phys. Rev. 41, 721 (1932).

[4] J. Krieger and C. Rosenzweig, Phys. Rev. 164, 171 (1967).

[5] J. Krieger, M. Lewis, and C. Rosenzweig, J. Chem. Phys. 47, 2942 (1967).

[6] R. Dutt, A. Khare, and U. P. Sukhatme, Phys. Lett. B 181, 295 (1986).

[7] R. Dutt, A. Khare, and U. Sukhatme, Am. J. Phys. 59, 723 (1991).

[8] F. Cooper, J. Ginocchio, and A. Khare, Phys. Rev. D 36, 2458 (1987).

[9] A. Adhikari, R. Dutt, A. Khare, and U. Sukhatme, Phys. Rev. A 38, 1679 (1988).

[10] L. E. Gendenshtein, Pis'ma Zh. Eksp. Teor. Fiz. 38, 299 (1983) [JETP Lett. 38, 356 (1983)]; L. E. Gendenshtein and I. V. Krive, Usp. Fiz. Nauk [Sov. Phys. Usp. 28, 645 (1985)].

[11] K. Raghunathan, M. Seetharaman, and S. S. Vasan, Phys. Lett. B 188, 351 (1987).

[12] D. Barclay and C. Maxwell, Phys. Lett. A 157, 357 (1991).

[13] Y. P. Varshni, J. Phys. A 25, 5761 (1992).

[14] C. X. Chuan, J. Phys. A 23, L659 (1992).
[15] Y. Ralchenko and V. Semenov, J. Phys. A 24, L1305 (1992).

[16] L. Boya, M. Kmiecik, and A. Bohm, Phys. Rev. D 35, 1255 (1987).

[17] A. Jevicki and J. Rodrigues, Phys. Lett. B 146, 55 (1984).

[18] R. Dutt, A. Gangopadhyaya, A. Khare, A. Pagnamenta, and U. Sukhatme, Phys. Lett. A 174, 363 (1993).

[19] R. Dutt, A. Khare, and U. Sukhatme, Am. J. Phys. 56, 163 (1988).

[20] J. W. Dabrowska, A. Khare, and U. Sukhatme, J. Phys. A 21, L195 (1988).

[21] R. De, R. Dutt, and U. Sukhatme, J. Phys. A 25, L843 (1992).

[22] A. Inomata and G. Junker (unpublished).

[23] O. L. De Lange and R. E. Raab, Operator Methods in Quantum Mechanics (Clarendon, Oxford, 1991).

[24] S. C. Miller, Jr. and R. H. Good, Jr., Phys. Rev. 91, 174 (1953).

[25] Y. Murayama, Phys. Lett. A 136, 455 (1989).

[26] U. Sukhatme and A. Pagnamenta, Am. J. Phys. 59, 944 (1991).

[27] A. Pagnamenta and U. Sukhatme, Phys. Lett. A 151, 7 (1990).

[28] R. Dutt, R. De, R. Adhikari, and A. Comtet, Phys. Lett. A 152, 381 (1990).

[29] U. Sukhatme and P. Panigrahi, UIC Report No. UICHEP-TH/92-7 (1992) (unpublished). 